\title{
ASPECTOS SEDIMENTOLÓGICOS E GEOQUÍMICOS DO TESTEMUNHO ANP 1040, DO TALUDE CONTINENTAL, PORÇÃO OESTE DO ESTADO DO CEARÁ
}

\author{
Agathe Arrissa Noucoucouk ${ }^{1}$ \\ George Satander Sá Freire ${ }^{2}$ \\ Robbyson Mendes Melo ${ }^{3}$ \\ Eldemar de Albuquerque Menor ${ }^{4}$ \\ Narelle Maia de Almeida ${ }^{2}$ \\ 10.18190/1980-8208/estudosgeologicos.v30n2p26-37 \\ ${ }^{1}$ Universidade Federal do Ceará -UFC - Programa de Pós-graduação em Geologia. \\ ${ }^{2}$ Universidade Federal do Ceará (UFC), Laboratório de Geologia Marinha e Aplicada, \\ Departamento de Geologia. \\ ${ }^{3}$ Universidade Federal de Pernambuco (UFPE), Laboratório de Micropaleontologia Aplicada \\ (LAGESE/LITPEG). \\ ${ }^{4}$ Universidade Federal de Pernambuco (UFPE), Departamento de Geologia.
}

\section{RESUMO}

O testemunho ANP 1040 analisado foi coletado a uma profundidade de $1900 \mathrm{~m}$ no oceano Atlântico Equatorial, distando de 100,8 km da linha de costa do município de Itapipoca, Ceará. Foram realizadas análises granulométricas, teor de carbonato de cálcio, teor de matéria orgânica, teor de nitrogênio total e geoquímica por fluorescência de raios-X dos elementos. Os dados mostram que o testemunho ANP 1040 é constituído pelas fácies lama, lama arenosa e areia lamosa, compostas por sedimentos bioclásticos classificados como vasa calcária. A diminuição da influência marinha na sedimentação foi constatada em intervalos intermediários do testemunho que apresentaram altos teores das razões de $\mathrm{Ti} / \mathrm{Ca}$ e $\mathrm{Fe} / \mathrm{Ca}$ que foram correlacionados aos valores elevados de lama, de matéria orgânica, e de baixa porcentagem do conteúdo carbonático. Os valores altos destas razões $(\mathrm{Ti} / \mathrm{Ca}$ e $\mathrm{Fe} / \mathrm{Ca}$ ) estão relacionados a um aumento da contribuição sedimentar continental, evidenciando períodos de maior precipitação na região, indicando um clima tropical úmido que aumentou o escoamento das drenagens continentais e, consequentemente, o suprimento de sedimentos terrestres para o talude continental. Além disso, os altos valores da razão Ti/Al encontrados na base do testemunho estão relacionados um aporte de sedimentos eólicos, enquanto valores baixos registrados no topo indicam uma proveniência de sedimento fluvial. Este trabalho contribui para o conhecimento da Geologia do Quaternário da sub-bacia Icaraí, Bacia do Ceará, Margem Equatorial Brasileira.

Palavras-Chave: Correlações paleoclimáticas; Razões Fe/Ca e Ti/Ca; Aporte terrígeno.

\begin{abstract}
The ANP 1040 core analyzed was collected at a depth of $1900 \mathrm{~m}$ in the Equatorial Atlantic Ocean, around $100.8 \mathrm{~km}$ from the coastline of the municipality of Itapipoca, Ceará. Particle size analyzes, geochemistry by X-ray fluorescence, calcium carbonate, organic matter and total nitrogen contents were performed. The data show that the ANP 1040 core contains the mud, sandy mud and muddy facies, composed of bioclastic sediments classified as limestone. The decrease of the marine influence on the sedimentation was observed in the intermediate interval of the core because it presented high levels of $\mathrm{Ti} / \mathrm{Ca}$ and $\mathrm{Fe} / \mathrm{Ca}$ ratios. In addition, this interval was correlated to high values of mud, organic matter, and low percentage of carbonate content. The high values of these ratios $(\mathrm{Ti} / \mathrm{Ca}$ and $\mathrm{Fe} / \mathrm{Ca}$ ) are related to an increase of the continental sedimentary contribution, denoting a period of a greater precipitation in the region, indicating a humid tropical climate that increased the flow of the continental drainages and, consequently, the supply of terrestrial
\end{abstract}


sediments to the continental slope. In addition, the high values of the Ti/Al ratio at the base of the core are related to a supply of eolian sediments, while low values of Ti/Al at the top of the core indicated a fluvial source. This work contributes to the knowledge of the Quaternary Geology of the Icaraí sub-basin, Ceará Basin, Brazilian Equatorial Margin.

Keywords: Paleoclimatic correlations; Fe/Ca and Ti/Ca ratios; Terrigenous contribution.

\section{INTRODUÇÃO}

Apesar do avanço no conhecimento da plataforma e talude continental do Ceará, dados da região do talude inferior da subbacia de Icaraí (Bacia do Ceará) são escassos. Estudos já realizados demonstram que o talude continental do Estado do Ceará é composto por sedimentos mistos (siliciclásticos e bioclásticos) com alto conteúdo carbonático e porcentagem baixa de matéria orgânica (Marques et al., 2007; Vidal et al., 2008; Nascimento et al., 2009, Lopes et al., 2017; Lins et al., 2018; Moraes et al., 2015). Os resultados dos trabalhos supracitados indicaram um retrabalhamento dos sedimentos pela ação das ondas, das marés e das correntes marinhas.

O conhecimento da composição e dos aspectos texturais dos sedimentos permite a compreensão das tendências naturais do ambiente, de forma que os parâmetros granulométricos constituem uma excelente ferramenta para avaliação da hidrodinâmica nos fundos de ambientes marinhos (Pettijohn, 1975). A distribuição do sedimento e sua granulometria, por sua vez, dependem do clima, da proximidade da fonte e da energia de ondas e correntes que irão moldá-los ao fundo, de acordo com os vários processos hidrodinâmicos (Nittrouer et al., 2007).
Dentro desse aspecto, tendo em vista um ambiente favorável à gênese de recursos minerais marinhos e a perspectiva da reconstrução paleoclimática, a caracterização sedimentológica e geoquímica do talude continental do Ceará é um importante recurso para entender a gênese e a dinâmica dos processos geológicos atuantes na região.

A área de estudo (Fig. 1) está inserida no contexto geológico da Sub-bacia Icaraí, Bacia do Ceará. A sub-bacia de Icaraí é localizada entre as sub-bacias de Acaraú e de Mundaú, sendo separada da Sub-bacia de Mundaú pela falha de Forquilha (Antunes et al., 2008), e da Sub-bacia de Acaraú pelo prolongamento norte do Lineamento Transbrasiliano. Seus limites norte e sul são o ramo sul da Zona de Fratura Romanche e a faixa de afloramento do embasamento cristalino respectivamente (Costa et al., 1990). O conteúdo sedimentar da Sub-bacia Icaraí é caracterizado pela deposição da sequência sedimentar marinha, composta por sedimentos transicionais, marinhos rasos e marinhos profundos do Membro Uruburetama da Formação Ubarana, e pelos sedimentos de talude do Membro Itapagé (Formação Ubarana) e pelos sedimentos plataformais das formações Tibau e Guamaré (Condé et al., 2007). 


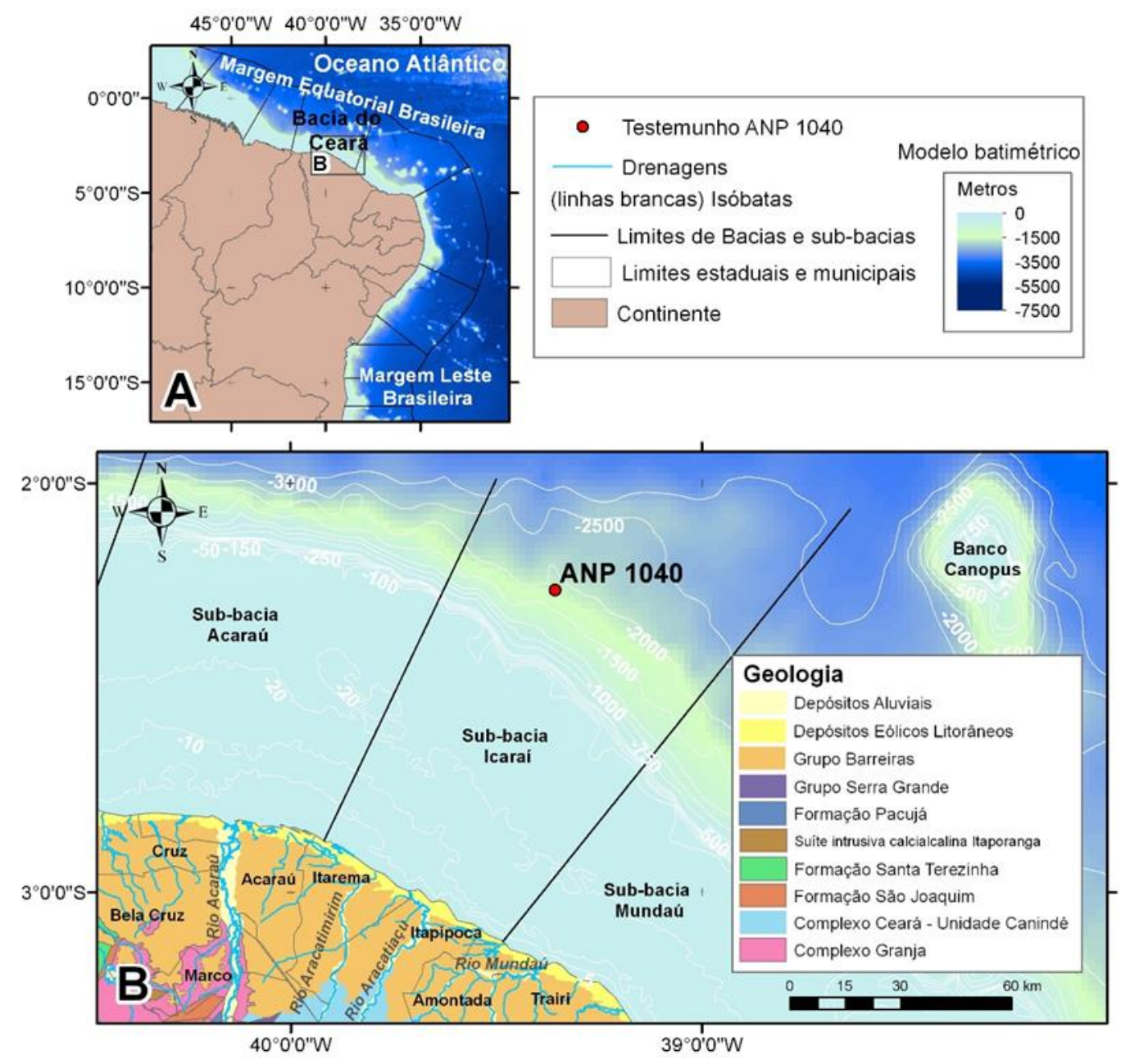

Figura 1 Localização da área de estudo, com destaque para a Bacia do Ceará na Margem Equatorial Brasileira (A) e o ponto de coleta do testemunho ANP 1040 na sub-bacia Icaraí, porção oeste do Estado do Ceará (B).

Desta forma, o presente trabalho tem como objetivo caracterizar os aspectos sedimentológicos e geoquímicos dos sedimentos do testemunho ANP 1040 coletado no talude continental da Sub-bacia Icaraí, tecendo considerações sobre sua proveniência e os aspectos ambientais.

\section{MATERIAL E MÉTODOS}

O testemunho ANP 1040 foi coletado entre as coordenadas $39^{\circ} 21^{\prime} 27^{\prime \prime}$ W e $2^{\circ} 15^{\prime} 40$ ' S pelo navio Fugro Odissey e cedido pela Agência Nacional do Petróleo, Gás Natural e BioCombustível (ANP) ao
Laboratório de Geologia Marinha e Aplicada (LGMA) da Universidade Federal do Ceará (UFC). O testemunho possui 1,68 $\mathrm{m}$ de espessura e foi coletado na porção Oeste da Bacia do Ceará (Sub-bacia de Icaraí) a uma distância de $100,8 \mathrm{~km}$ do litoral do município de Itapipoca-CE, a $1900 \mathrm{~m}$ de profundidade (Fig. 1). A seção foi descrita baseada em suas características sedimentológicas, como suas variações de cores (utilizando Geological Rock-Color Chart), estruturas, contatos e tamanho de grão. Para as análises realizadas, foram utilizadas 15 amostras, coletadas em um intervalo amostral de $10 \mathrm{~cm}$. 
$\mathrm{Na}$ análise granulométrica, a amostra foi lavada com água corrente sobre uma peneira com malha de abertura de 0,062 $\mathrm{mm}$. As frações silte e argila foram coletadas em um recipiente e deixado em repouso por um período de 48 horas para decantação, sendo posteriormente levadas à estufa $\left(60^{\circ} \mathrm{C}\right) . \mathrm{O}$ material retido na peneira de $0,062 \mathrm{~mm}$ também foi levado à estufa $\left(60^{\circ} \mathrm{C}\right)$ e, após secagem, foi realizado peneiramento a seco em um sistema tipo ROT-AP, utilizando um jogo de peneiras que variam de $4 \mathrm{~mm}$ até $0,062 \mathrm{~mm}$. O material retido em cada peneira foi coletado e pesado para cálculos percentuais, incluindo a fração cascalho (grãos com diâmetro $>2 \mathrm{~mm}$ ), a fração areia (2 a 0,062 $\mathrm{mm}$ ) e a fração lama (sedimentos finos, com diâmetro < 0,062 mm).

Além da análise granulométrica foram determinados nos sedimentos totais, os teores de carbonato de cálcio (método do calcímetro de Bernand modificado), de matéria orgânica (método de WalkleyBlack modificado), e de nitrogênio total (método de Kjeldahl por via úmida). Ademais, análises de fluorescência de Raios-X também foram realizadas utilizando a infraestrutura do Departamento de Física da Universidade Federal do CearáUFC.

Para classificar a granulometria dos sedimentos foi utilizado o diagrama triangular de Shepard (1954), que inclui os percentuais das frações cascalho, areia e lama. Por fim, os dados obtidos foram tratados utilizando os softwares ArcGis 10.3, SedLog 3.0, ANASED 5.0 e Corel Draw X3.

\section{RESULTADOS}

Macroscopicamente, o testemunho ANP 1040 é composto por areia e lama (silte + argila). Possui estrutura homogênea, com presença de conchas (Fig. 2).

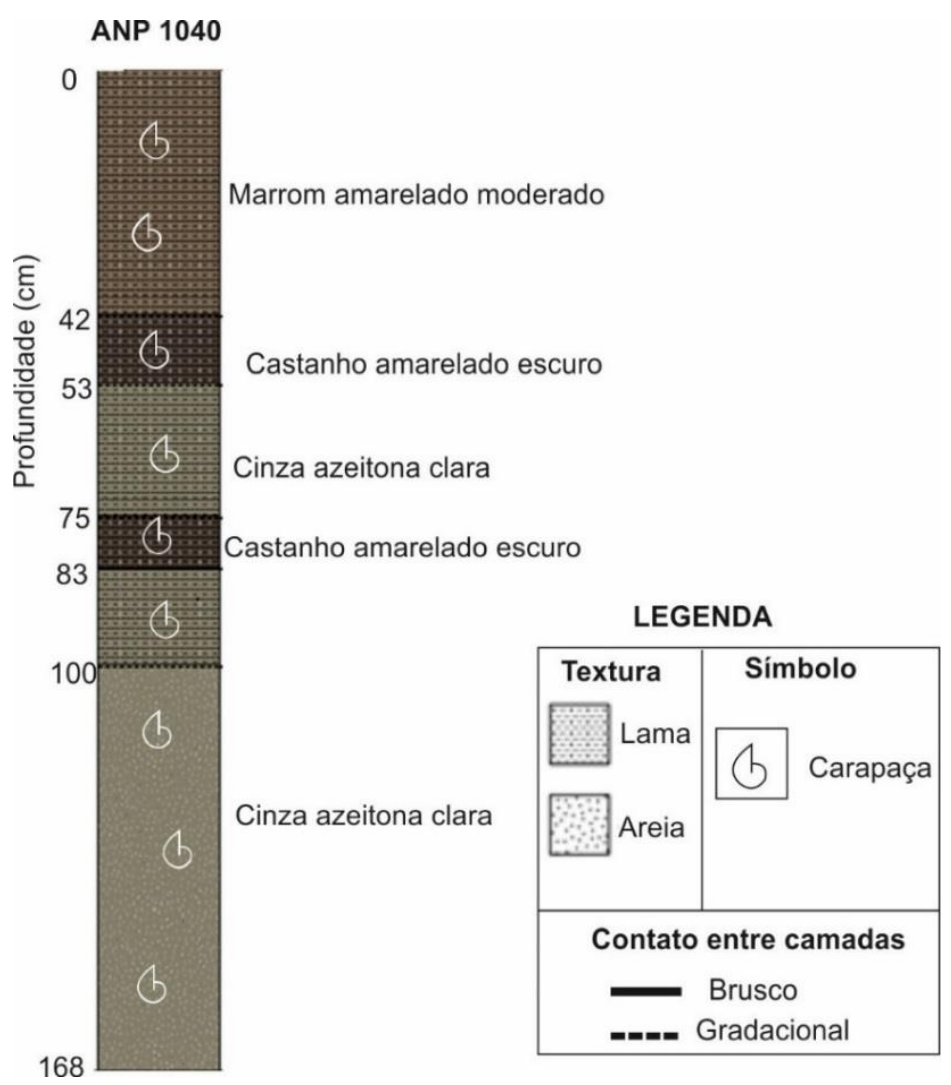

Figura 2 Perfil litológico do testemunho ANP 1040. 


\section{ASPECTOS SEDIMENTOLÓGICOS E GEOQUÍMICOS DO TESTEMUNHO ANP 1040...}

Os resultados das análises granulométricas (Fig. 3) mostram que a fração cascalho é a menos abundante no testemunho, com média de $0,21 \%$, variando de $0 \%$ a $1,08 \%$. Seu maior valor ocorre na amostra 140-141 cm do testemunho. A média dos valores da fração areia é de $41,50 \%$, variado entre $7,83 \%$ e $70,96 \%$. Seu maior valor está na base do testemunho, a partir de $120 \mathrm{~cm}$. A média dos teores de lama (silte e argila) é de $58,29 \%$, e sua variação está compreendida entre $28,44 \%$ e $92,17 \%$, com maior valor na porção mediana do testemunho, entre 79 e $92 \mathrm{~cm}$. A granulometria dos sedimentos apresentou heterogeneidade marcante, onde os menores teores de sedimentos finos (silte + argila) foram observados da base até o nível de $100 \mathrm{~cm}$.

De acordo com a classificação dos sedimentos no diagrama de Shepard (1954), as amostras foram classificadas como lama (amostras: $9-11 \mathrm{~cm}, 39-41 \mathrm{~cm}, 50-52 \mathrm{~cm}$, $79-81 \mathrm{~cm}$ e $89-92 \mathrm{~cm})$, lama arenosa (amostras: 19-21 cm, 30-32 cm, 60-62 cm, e 69-71 cm) e areia lamosa (amostras: 100$101 \mathrm{~cm}, 110-111 \mathrm{~cm}, 120-121 \mathrm{~cm}, 130-131$ $\mathrm{cm}, 140-141 \mathrm{~cm}$ e $150-151 \mathrm{~cm}$ ).

A análise de carbonato de cálcio $\left(\mathrm{CaCO}_{3}\right)$ revelou que o testemunho estudado é composto por um sedimento bioclástico classificado como vasa calcária (Vital et al. 2005), pois todas as amostras possuem teor de $\mathrm{CaCO}_{3}$ acima de $70 \%$ e teor de lama acima de $15 \%$. O testemunho apresenta uma média de $98,64 \%$ de $\mathrm{CaCO}_{3}$ com uma redução marcante registrada na amostra $79-81 \mathrm{~cm}$, chegando a 92,42\% (Fig. 3).

A média dos teores de matéria orgânica é $1,5 \%$, com valores entre $0,9 \%$ e $2,2 \%$, onde na amostra 79-81 cm encontrase o maior dentre estes (Fig. 3).

O Nitrogênio total (NT) apresentou uma porcentagem média de $0,03 \%$, onde no testemunho ocorre uma variação de $0,01 \%$ a $0,05 \%$ (Fig. 3).

Os dados da razão carbono e nitrogênio $(\mathrm{C} / \mathrm{N})$ apresentou uma média de 40,1 com uma variação de 17,0 a 80,0 . Os seus altos teores estão contidos na base do testemunho, onde na amostra $110-111 \mathrm{~cm}$ encontra-se o maior dentre estes valores (Fig. 3). A dispersão entre o carbono e o nitrogênio total apresentou uma correlação muito fraca de $\mathrm{R}=0,36$.

Os teores dos elementos químicos do testemunho ANP 1040 variaram ao longo da sucessão sedimentar, principalmente o cálcio, silício, ferro e alumínio (Tabela 1).

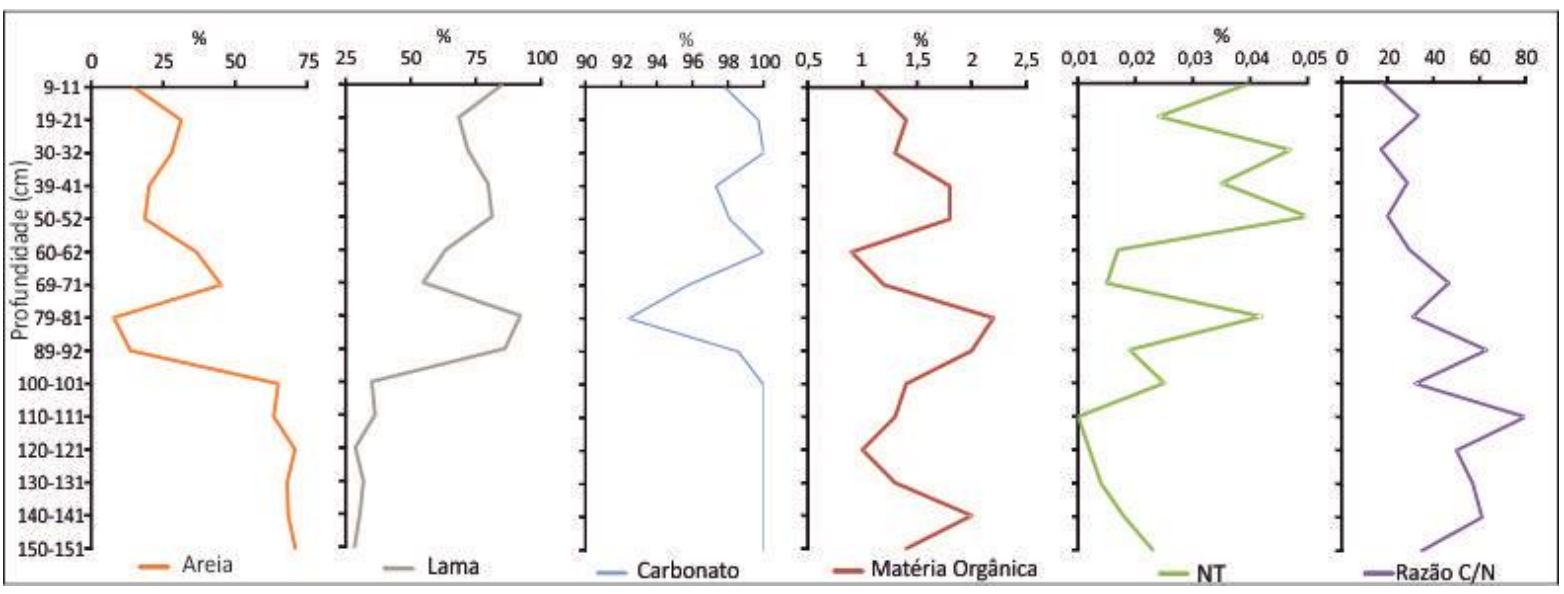

Figura 3 Valores percentuais de areia, lama, teores de carbontato de cálcio, matéria orgânica e nitrogênio total (NT), além da razão carbono/nitrogênio (C/N) no testemunho ANP 1040. 
Tabela 1 Elementos químicos encontrados ao longo do testemunho ANP 1040.

\begin{tabular}{|c|c|c|c|c|c|c|c|c|c|c|c|c|c|c|c|c|c|}
\hline $\begin{array}{c}\text { Amostras } \\
(\mathbf{c m})\end{array}$ & $\begin{array}{l}\mathrm{Ca} \\
\%\end{array}$ & $\begin{array}{l}\mathrm{Si} \\
\%\end{array}$ & $\begin{array}{l}\mathrm{Fe} \\
\%\end{array}$ & $\begin{array}{l}\text { Al } \\
\%\end{array}$ & $\begin{array}{l}\mathbf{K} \\
\%\end{array}$ & $\begin{array}{l}\mathrm{Cl} \\
\%\end{array}$ & $\begin{array}{l}\mathbf{P} \\
\%\end{array}$ & $\begin{array}{l}\mathrm{Sr} \\
\%\end{array}$ & $\begin{array}{l}\mathrm{Ti} \\
\%\end{array}$ & $\underset{\%}{M n}$ & $\begin{array}{l}\mathrm{Zn} \\
\%\end{array}$ & $\begin{array}{c}\text { Cd } \\
\%\end{array}$ & $\begin{array}{l}\mathbf{S} \\
\%\end{array}$ & $\begin{array}{c}\text { Rb } \\
\%\end{array}$ & $\begin{array}{l}\mathrm{V} \\
\%\end{array}$ & $\begin{array}{l}\mathrm{Br} \\
\%\end{array}$ & $\begin{array}{l}\mathrm{Na} \\
\%\end{array}$ \\
\hline 9-11 & 34,9 & 20,4 & 26,5 & 10,2 & 2,7 & 0,6 & 0,2 & 0,8 & 2,0 & 1,6 & & & 0,1 & & & & \\
\hline $19-21$ & 49,7 & 20,4 & 15,9 & 8,1 & 3,0 & 0,3 & 0,2 & 0,8 & 1,6 & & & & & & & & \\
\hline $30-32$ & 40,8 & 21,5 & 20,8 & 9,5 & 3,3 & 1,2 & & 0,8 & 1,4 & 0,2 & 0,1 & 0,6 & & & & & \\
\hline $39-41$ & 28,6 & 27,2 & 27,8 & 9,7 & 3,9 & 0,4 & & 0,5 & 1,7 & 0,3 & & & & & & & \\
\hline $50-52$ & 26,3 & 28,0 & 27,8 & 10,1 & 3,9 & 0,5 & 0,2 & 0,5 & 2,0 & 0,2 & & & 0,2 & & 0,3 & & \\
\hline $60-62$ & 41,7 & 22,6 & 12,3 & 7,7 & 3,7 & 0,4 & 0,1 & 0,6 & 1,5 & 0,3 & 0,1 & & 0,1 & & & & \\
\hline $69-71$ & 40,1 & 20,4 & 23,2 & 8,9 & 3,1 & 0,6 & 0,2 & 0,6 & 2,5 & 0,3 & 0,1 & & 0,1 & & & & \\
\hline $79-81$ & 18,6 & 30,0 & 31,3 & 11,3 & 4,7 & 0,7 & & 0,3 & 2,8 & 0,2 & & & & 0,1 & & & \\
\hline $89-92$ & 19,1 & 31,1 & 30,8 & 11,1 & 4,4 & 0,4 & & 0,3 & 2,3 & 0,2 & 0,1 & & & 0,1 & & & \\
\hline $100-101$ & 49,1 & 19,3 & 16,4 & 7,5 & 3,2 & 1,5 & 0,2 & 0,8 & 1,6 & 0,5 & & & 0,1 & & & & \\
\hline $110-111$ & 48,7 & 17,6 & 18,3 & 7,3 & 3,1 & 1,9 & 0,2 & 0,8 & 1,9 & & 0,1 & & 0,1 & & & & \\
\hline $120-121$ & 49,8 & 17,5 & 16,3 & 5,9 & 3,5 & 4,6 & & 0,7 & 1,3 & 0,3 & & & & & & & \\
\hline $130-131$ & 52,5 & 16,5 & 17,8 & 5,7 & 3,1 & 1,9 & & 0,7 & 1,6 & 0,3 & & & 0,1 & & & & \\
\hline $140-141$ & 51,1 & 16,5 & 18,9 & 5,7 & 3,1 & 20,2 & & 0,6 & 1,6 & 0,3 & & & 0,2 & & & & \\
\hline $150-151$ & 31,7 & 15,4 & 13,4 & 5,7 & 4,7 & 17,4 & & 0,4 & 1,2 & 0,2 & 0,1 & & 0,6 & & & 0,3 & 8,9 \\
\hline
\end{tabular}

Dentre as razões dos elementos químicos analisados (Fig. 4), as razões $\mathrm{Ti} / \mathrm{Ca}$ e $\mathrm{Fe} / \mathrm{Ca}$ apresentaram um comportamento semelhante, com altos teores concentrados na porção mediana do testemunho (amostras 79-81 cm e 89-92 $\mathrm{cm})$. Os valores foram relativamente constantes da base até a amostra 100-101 $\mathrm{cm}$, onde houve uma tendência geral de aumento, chegando até 0,15 para $\mathrm{Ti} / \mathrm{Ca} \mathrm{e}$ 1,68 para $\mathrm{Fe} / \mathrm{Ca}$ na amostra $79-81 \mathrm{~cm}$. A partir de então, há uma queda nos valores destas razões até a amostra 60-62 cm com 0,03 para $\mathrm{Ti} / \mathrm{Ca}$ e 0,3 para $\mathrm{Fe} / \mathrm{Ca}$.
A razão $\mathrm{Fe} / \mathrm{K}$ (Fig. 4) apresentou vários picos de acréscimo e decréscimo ao longo do testemunho, com valor máximo de 9,7 na amostra 9-11 cm e mínimo de 2,85 na amostra 150-151. Da base para o topo, os valores da razão Ti/Al (Fig. 4) aumentaram até 0,28 na amostra $130-131 \mathrm{~cm}$ e, de uma forma geral, diminuíram em direção ao topo na amostra $30-32 \mathrm{~cm}(0,14)$, com um pico discordante devido a um alto valor da amostra $69-71 \mathrm{~cm}$. Os maiores valores desta razão foram registrados na base do testemunho. 


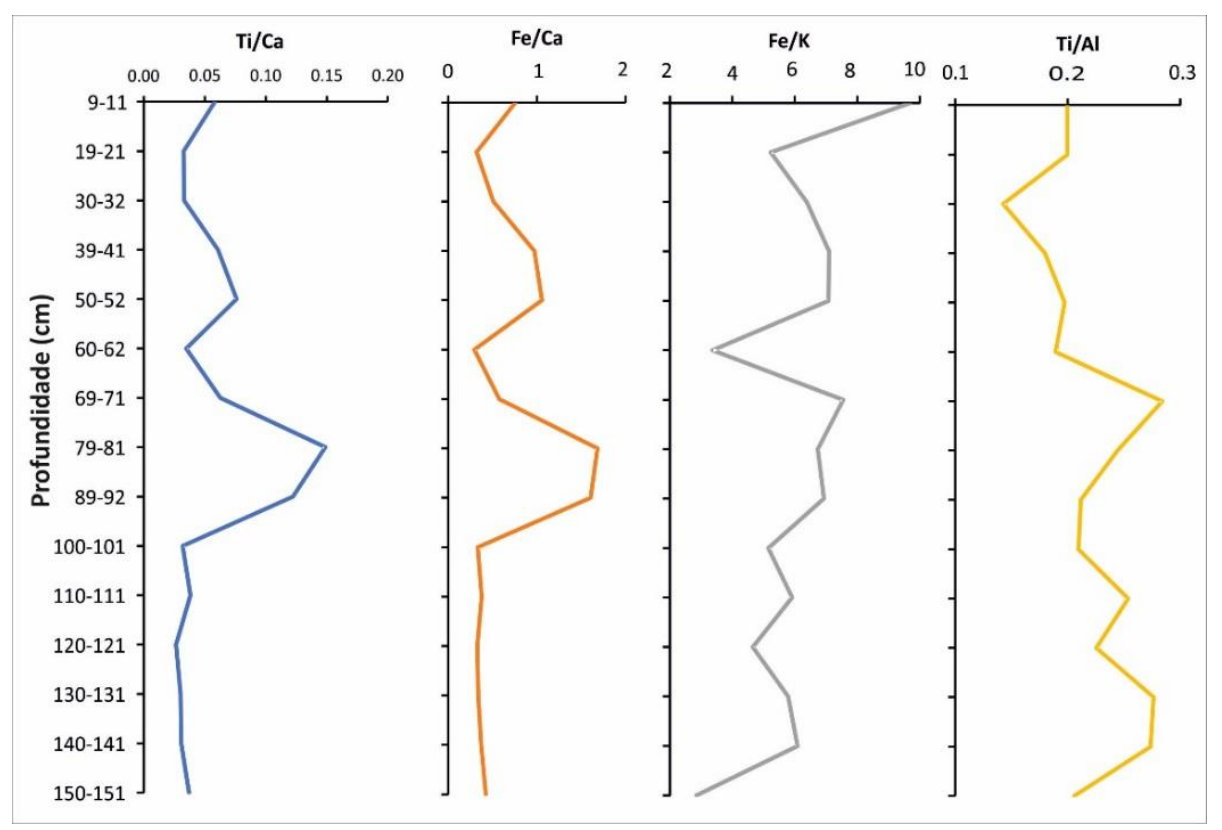

Figura 4 Razões elementares de Ti/Ca, $\mathrm{Fe} / \mathrm{Ca}, \mathrm{Fe} / \mathrm{K}$ e Ti/Al do testemunho ANP 1040.

De forma a amenizar as incertezas geradas pelos problemas associados à utilização dos metais (mudanças na produtividade biológica marinha que afetam a interpretação de concentrações de elementos únicos), foram realizadas as dispersões entre $\mathrm{Fe}$ e $\mathrm{Ca}, \mathrm{Al}$ e $\mathrm{Ca}, \mathrm{Al}$ e Fe,
$\mathrm{K}$ e $\mathrm{Ca}, \mathrm{K}$ e Fe, $\mathrm{K}$ e Al, Ti e Ca, Ti e Fe, Ti e Al, e Ti e K. Os elementos apresentaram boas correlações para as amostras do testemunho com exceção de $\mathrm{K}$ e $\mathrm{Fe}, \mathrm{K}$ e Al, e Ti e K (Tabela 2A). Também foram feitas as correlações entre as razões $\mathrm{Ti} / \mathrm{Ca}, \mathrm{Fe} / \mathrm{Ca}$, $\mathrm{Fe} / \mathrm{K}$ e Ti/Al (Tabela 2B).

Tabela 2 Resultados das correlações dos elementos e suas razões no testemunho ANP 1040: A. Dispersões entre os elementos $\mathrm{Ca}, \mathrm{Fe}, \mathrm{Al}, \mathrm{K}$ e Ti; B. Dispersões entre as razões $\mathrm{Ti} / \mathrm{Ca}, \mathrm{Fe} / \mathrm{Ca}$, $\mathrm{Fe} / \mathrm{K} \mathrm{e} \mathrm{Ti/Al.}$

A

\begin{tabular}{c|cccc} 
& $\mathbf{C a}$ & $\mathbf{F e}$ & $\mathbf{A l}$ & $\mathbf{K}$ \\
$\mathbf{F e}$ & $-0,77$ & & & \\
$\mathbf{A l}$ & $-0,78$ & 0,85 & & \\
$\mathbf{K}$ & $-0,77$ & 0,32 & 0,29 & \\
$\mathbf{T i}$ & $-0,59$ & 0,79 & 0,73 & 0,20
\end{tabular}

B

\begin{tabular}{c|ccc}
\hline & $\mathbf{T i} / \mathbf{C a}$ & $\mathbf{F e} / \mathbf{C a}$ & $\mathbf{F e} / \mathbf{K}$ \\
$\mathbf{F e} / \mathbf{C a}$ & 0,97 & & \\
$\mathbf{F e} / \mathbf{K}$ & 0,43 & 0,49 & \\
$\mathbf{T i} / \mathbf{A l}$ & 0,07 & $-0,08$ & 0,07
\end{tabular}

\section{DISCUSSÃO}

O testemunho estudado possui as fácies lama, lama arenosa e areia lamosa (Shepard, 1954). Cada fração granulométrica (areia + lama) corresponde a um transporte de alta ou baixa energia, quando sujeitas a diferentes ações de correntes. Observou-se que a fração areia predominou da base até a profundidade 100 $\mathrm{cm}$ indicando um transporte de maior energia durante a deposição deste intervalo, 
enquanto a maior porcentagem das frações lamosas esteve contida na porção intermediária, significando o predomínio de um transporte por suspensão de baixa energia.

Em relação ao pico menor de $\mathrm{CaCO}_{3}$, localizado na porção intermediária do testemunho ANP 1040 (Fig. 3), pode corresponder ao período de grande aporte de material terrígeno na área de estudo. No Oceano Atlântico, durante os períodos interglaciais o teor de carbonato dos sedimentos do fundo do mar é maior do que durante os períodos glaciais, diferente do que ocorre no Oceano Índico e Pacífico (Volat et al., 1980; Hodell, et al., 2001). As explicações para essas diferenças no tempo e no local foram objeto de extensa discussão (Suess, 1956; Volat et al., 1980). Os principais fatores que levam a essa variabilidade são a taxa de produção de carbonato de cálcio em relação à matéria orgânica, o estado de saturação da água do mar profunda e a taxa de entrada de material não carbonatado.

Observou-se na área de estudo, que os valores de matéria orgânica são mais controlados pela presença de lamas do que pela profundidade. Maiores concentrações de matéria orgânica estão associadas a áreas com baixa hidrodinâmica junto ao fundo e ao maior teor de sedimentos finos (Oliveira et al., 2014). Esta correlação entre sedimentos finos e matéria orgânica, já foi observada em diversos trabalhos (Barcellos et al., 2011; Santos, 2016; Oliveira, 2016), e indica um ambiente de baixa energia com contribuição de partículas de origem orgânica.

Os dados de Nitrogênio total obtidos neste trabalho concordam com estudos já desenvolvidos no talude continental cearense e em outros taludes da margem equatorial brasileira (Lima Neto, 2017; Lins, 2018; Lopes, 2018), em que os teores de nitrogênio são baixos, com resultados inferiores a $0,2 \%$. A natureza do nitrogênio (orgânico ou inorgânico) pode ser verificada através da plotagem dos teores de carbono contra os teores de nitrogênio.
Segundo Fernandes et al. (2011), se o carbono e o nitrogênio nos sedimentos são gerados a partir da mesma fonte, estes devem apresentar uma boa correlação. Uma forte correlação linear positiva confirma a natureza orgânica do nitrogênio e valida a utilização da razão $\mathrm{C} / \mathrm{N}$ para avaliar a fonte da matéria orgânica sedimentar (Lourenço, 2007), o que não foi o caso do testemunho ANP 1040, já que o mesmo apresentou uma correlação linear muito fraca, sendo que os valores da razão $\mathrm{C} / \mathrm{N}$ podem ser subestimados, em função do nitrogênio não ser de origem essencialmente orgânica. Nesse caso, a razão $\mathrm{C} / \mathrm{N}$ não pode ser utilizada como elemento para caracterizar as fontes da matéria orgânica. A razão $\mathrm{C} / \mathrm{N}$ tanto para fitoplâncton quanto para zooplâncton fornece em média um valor em torno de 6 enquanto essa razão para material terrígeno fornece valores em torno de 20 ou mais (Meyers, 1997). Os teores elevados da razão $\mathrm{C} / \mathrm{N}$ ao longo dos testemunhos podem estar relacionados à degradação parcial da matéria orgânica algal (Lourenço, 2007).

Os valores altos das razões $\mathrm{Ti} / \mathrm{Ca}$ e $\mathrm{Fe} / \mathrm{Ca}$ encontrados na porção intermediária do testemunho estudado estão relacionados a um aumento da drenagem continental, evidenciando períodos de maior precipitação na região do nordeste do Brasil como consequência, por exemplo, do deslocamento da Zona de Convergência InterTropical (ZCIT) para o norte (Zhang et al., 2017). Tanto o Ti quanto o Fe são fornecidos a partir da bacia hidrográfica da região adjacente e estão associados aos compontentes terrígenos (Nagai, 2009), enquanto o $\mathrm{Ca}$ é um elemento relacionado os organismos calcários, como foraminíferos, ostracodes, moluscos, e cocolitoforídeos no oceano, portanto, está associado à produtividade biogênica marinha da plataforma e talude continental (Depetris et al., 2003; Govin et al., 2012; Razik et al., 2013). O aumento episódico dos marcadores terrígenos pode ter sido causado por mudanças climáticas nas áreas costeiras do nordeste do Brasil (Rao et al., 1993). Um clima ligeiramente mais úmido 
e maiores taxas de precipitação nas bacias hidrográficas dos rios costeiros aumentariam a erosão da terra, o escoamento dos rios e, assim, aumentariam o suprimento de sedimentos terrestres para o talude continental superior (Arz et al., 1998).

Os valores altos de Ti/Al encontrados na base do testemunho estão relacionados a um aporte de sedimentos eólicos, enquanto valores baixos registrado no topo indicam uma proveniência de sedimento fluvial (Govin et al., 2012). Em relação à distribuição de $\mathrm{Fe} / \mathrm{K}$, os autores supracitados afirmaram que ao longo das margens continentais do Atlântico os altos valores de $\mathrm{Fe} / \mathrm{K}$ em sedimentos africanos e brasileiros de superfície estão relacionados com áreas tropicais indicando a entrada dominante de material altamente intemperizado, enquanto os baixos valores de $\mathrm{Fe} / \mathrm{K}$ são provenientes de regiões mais secas, e refletem a entrada de material pouco intemperizado quimicamente. No testemunho estudado, observou-se uma correlação muita fraca entre os elemenos $\mathrm{K}$ e Fe, $\mathrm{K}$ e $\mathrm{Al}$ e $\mathrm{Ti}$ e $\mathrm{K}$, bem como entre as correlações das razões elementares de $\mathrm{Fe} / \mathrm{K}$ e $\mathrm{Ti} / \mathrm{Ca}$ e $\mathrm{Fe} / \mathrm{K}$ e $\mathrm{Fe} / \mathrm{Ca}$, dificultando a possibilidade de maior precisão no uso da razão $\mathrm{Fe} / \mathrm{K}$ na área de estudo. Diferentemente, as razões $\mathrm{Ti} / \mathrm{Ca}, \mathrm{Fe} / \mathrm{Ca}$ e $\mathrm{Ti} / \mathrm{Al}$ se mostraram eficientes, possibilitando maior precisão na determinação dos aportes terrígenos na área de estudo, apontando uma fonte eólica predominante na base do testemunho e uma fonte fluvial no topo do mesmo. Alguns trabalhos realizados na margem equatorial nordestina do Brasil (Arz et al., 1998, 1999; Jaeschke et al., 2007), além de dados dos tributários da Amazônia (Bouchez et al., 2011) indicam também uma clara relação entre as razões $\mathrm{Fe} / \mathrm{Ca}$, Ti/Ca e o aporte continental para a bacia oceânica.

\section{CONCLUSÕES}

Em termos sedimentológicos, os resultados obtidos indicaram uma estrutura homogênea, com as fácies sedimentares distribuídas entre lama, lama arenosa e areia lamosa. Foram observados teores mais elevados de areia na base do testemunho, indicando um transporte sedimentar mais efetivo em comparação ao topo e ao meio. De acordo com os teores de carbonato, todos os sedimentos do testemunho foram classificados como bioclásticos, mais precisamente como vasa calcária. Já os valores de matéria orgânica na área de estudo são mais controlados pela presença de lama do que pela profundidade.

As dispersões entre carbono e nitrogênio não forneceram uma boa correlação, sugerindo que o nitrogênio dos sedimentos estudados pode não ser de origem essencialmente orgânica.

$\mathrm{O}$ aumento do aporte continental ou diminuição da influência marinha foi constatado pelos teores altos de $\mathrm{Ti} / \mathrm{Ca} \mathrm{e}$ $\mathrm{Fe} / \mathrm{Ca}$ que foram correlacionados aos valores elevados de lama, de matéria orgânica, e à baixa porcentagem do conteúdo carbonático. Essas características foram observadas, sobretudo, na porção intermediária do testemunho. Além disso, os valores baixos da razão $\mathrm{Ti} / \mathrm{Al}$ foram interpretados como sedimentos com influência de fonte fluvial, enquanto que os valores altos desta razão estariam relacionados a fonte eólica.

\section{Agradecimentos}

O presente trabalho foi realizado com apoio da Coordenação de Aperfeiçoamento de Pessoal de Nível Superior - Brasil (CAPES) - Código de Financiamento 001. Os autores agradecem ao Laboratório de Geologia Marinha e Aplicada (LGMA) do Departamento de Geologia da Universidade Federal do Ceará pela infraestrutura disponibilizada e ao Laboratório de Raios$\mathrm{X}$ do Departamento de Física desta mesma universidade pela realização das análises de fluorescência de raios- $X$. 


\section{REFERÊNCIAS}

Antunes, A.F.; Jardim, E.F.S., Araújo, R.G. da S.; Lima Neto, F.F. 2008. Caracterização tectonoestrutural do Campo de Xaréu (Sub-Bacia de Mundaú, Bacia do Ceará - NE do Brasil): abordagem multiescala e pluriferramental. Revista Brasileira de Geociências, 38(1), 88-105.

Arz, H.W.; Pätzold, J.; Wefer, G. 1998. Correlates millennial-scale changes in surface hydrography and terrigenous sediment yield inferred from lastglacial marine deposits off Northeastern Brazil. Quartenary Research, 50, 157-166.

Arz, H.W.; Pätzold, J. \& Wefer, G. 1999. Climatic changesthe last deglaciation recorded in sediment cores from the northeastern Brazilian continental margin, Geo-Marine Letters, 19(3), 209-218.

Barcellos, R.L.; Coelho JR., C.; Lins, S.R.; Silva, M.S.; Camargo, P.B.; Travassos, P.E. 2011. Island beaches morphological and sedimentary short term variations the case of SE Fernando de Noronha Island, South Atlantic, Brazil. Revista da Gestão Costeira Integrada, 11 (4): 471-478.

Bouchez, J. ; Gaillardet, J.; France-lanord, C.; Maurice, L. \& Dutra-Maia, P. 2011. Grain size control of river suspended sediment geochemistry: Clues from Amazon River depth profiles, Geochemistry Geophysics Geosystems, 12, Q03008.

Condé, V.C.; Lana, C.C.; Pessoa neto, O.C.; Roesner, E.H.; Morais Neto, J.M.; Dutra, D.C. 2007. Bacia do Ceará. Boletim de Geociências da Petrobras, Rio de Janeiro. 15(2), 347-355.

Costa, I.G.; Beltrami, C.V. \& Alves, L.E.M. 1990. A evolução tectono-sedimentar e o "habitat" do óleo na Bacia do Ceará. Boletim Geociências Petrobras, 4, 65-74.

Depetris, P.J.; Probst, J.L.; Pasquini, A.I. \& Gaiero, D.M. 2003. The geochemical characteristics of the Paraná River suspended sediment load: An initial assessment, Hydrological. Processes, 17(7), 1267-1277.

Govin, A.; Holzwarth, U.; Heslop, D.; Ford keeling, L.; Zabel, M.; Mulitza, S.; Collins, J.A.; \& Chiessi, C.M. 2012. Distribution of major elements in Atlantic surface sediments $\left(36^{\circ} \mathrm{N}-\right.$ $49^{\circ} \mathrm{S}$ ): Imprint of terrigenous input and continental weathering, Geochemistry Geophysics Geosystems, 13, Q01013.

Hodell, D.A.; Charles, C.D. \& Sierro, F.J. 2001. Late Pleistocene evolution of the ocean's carbonate system. Earth and Planetary Science Letters, 192, 109-124.

Jaeschke, A.; Rühlemann, C.; Arz, H.; Heil, G.; \& Lohmann, G. 2007. Coupling of millennial-scale changes in sea surface temperature and precipitation off northeastern Brazil withhighlatitude climate shifts during the last glacial period.

Lima Neto, O.I. 2017. Fortaleza, Análise textural e geoquímica dos sedimentos do talude continental do Oeste do Ceará. Departamento de Geologia, Universidade Federal do Ceará, Disertação de Mestrado, 104p.

Lins, J.P.C. 2018. Fortaleza, Caracterização sedimentológica Quaternária do talude continental do município de Luís Correia, Piauí. Departamento de Geologia, Universidade Federal do Ceará, Dissertação de Mestrado, 91p. Lins, J.P.C.; Lopes, J.P.F. \& Freire, G.S.S. $2018 . \quad$ Caracterização Sedimentológica do Poço SIS 720 Talude Continental Quaternário do Litoral do Piauí. Estudos geológicos (UFPE), 27, 15-27.

Lopes, J.P.F.; Lins, J.P.C. \& Freire, G.S.S. 2017. Sedimentologia do Testemunho ANP 1285 - Talude Continental de Luís Correia, Piauí. Estudos geológicos (UFPE), 27, 1-11.

Lopes, J.P.F. 2018. Fortaleza, Sedimentologia do talude continental 
frente a Luís Correia, Piauí. Departamento de Geologia, Universidade Federal do Ceará, Dissertação de Mestrado, 88p.

Lourenço, R.A. 2007. Aplicação de marcadores orgânicos moleculares em estudos oceanográficos e paleoceanográficos: Estudo de caso na margem continental superior do Sudeste do Brasil. Instituto Oceanográfico, Universidade de São Paulo, Tese do Doutorado. 158p.

Marques, W.S.; Menor, E.A.; Sial, A.N.; Manso, V. do A.V. \& FREIRE, G.S.S. 2006. Oceanographic parameters in continental margin of the state of Ceará (Northeastern Brazil) deduced from $\mathrm{C}$ and $\mathrm{O}$ isotopes in foraminifers. Anais da Academia Brasileira de Ciências, 79, 1-11.

Moraes, M.V.A.R.; Manso, V. do A.V. \& Freire, G.S.S. 2015. Morfologia e Sedimentologia da Plataforma Continental Interna do Município de Acaraú, Ceará, Brasil. revista de geociências do Nordeste, 1, 1-21.

Meyers, P.A. 1997. Organic geochemical proxies of paleoceanographic, paleolimnologic, and paleoclimatic processes. Organic Geochimistry, 27, 213-250.

Nagai, R.H. 2009. Variações de paleoprodutividade na plataforma de Cabo Frio RJ, durante o Holoceno. Dissertação de Mestrado. Instituto Oceanográfico, Universidade de São Paulo, 130p.

Nascimento, F.S.; Freire, G.S.S. \& Miola, B. 2009. Caracterização dos Granulados da Plataforma Continental do Nordeste do Brasil. Revista de Geologia (Fortaleza), 22, 15-26.

Nittrouer, C.A., Austin, J.A., Field, M.E., Kravitz, J.H., Syvitski, J.P.M., Wiberg, P.L., editors. 2007. Continental margin sedimentation: from sediment transport to sequence stratigraphy. Malden: Blackwell
Publishing. International Association of Sedimentologists. Special Publication, 37.

Oliveira, L.E.E. 2016. Recife, Estudo da matéria orgânica sedimentar como indicador ambiental nas adjacências do porto do recife, ilha deitamaracá e arquipélago de Fernando de Noronha (Pernambuco).

Instituto Oceanográfico, Universidade Federal de Pernambuco, Dissertação de Mestrado, 187p.

Oliveira, T.S.; Barcellos, R.L.; Schettini, C.A.F. \& Camargo, P.B. 2014. Processo sedimentar atual e distribuição da matéria orgânica em um complexo estuarino tropical, Recife, PE, Brasil. Journal of Integrated Coastal Zone Management, 14, 399-411.

Pettijohn, F.J. 1975. Sedimentary rocks. $3^{\mathrm{a}}$ ed. New York: Harper e row, 628 p.

Rao, V.B.; De Lima, M.C. \& Franchito, S.H. 1993. Seasonal and interannual variations of rainfall over eastern Northeast Brazil. Journal of Climate, 6, 1754-1763.

Razik, S.; Chiessi, C.M.; Romero, O.E. \& Von Dobeneck, T. 2013. Interaction of the South American monsoon system and the Southern Westerly wind belt during the last $14 \mathrm{kyr}$, Palaeogeography Palaeoclimatology Palaeoecology, 374, 28-40.

Santos, L.D. 2016. Recife, Dinâmica sedimentar e o comportamento sazonal da matéria orgânica no estuário do Rio Formoso - PE. CTG, Universidade Federal de Pernambuco, Dissertação de Mestrado, 98p.

Shepard, F.P. September, 1954. Nomenclature based on sand-silt-clay ratios. Journal Sedimentary Petrology. USA, 24, 151-158.

Suess, H.E. 1956. Absolut chronology of the last glaciation. Science, 123, 355357.

Vidal, R.M.B.; Becker, H.; Freire, G.S.S. 2008. Geoquímica de sedimentos da 
Plataforma Continental do Estado do Ceará - Brasil. Revista de Geologia (Fortaleza), 21, 223-231.

Zhang, Y.; Chiessi, C.M., Mulitza, S.; Sawakuchi, A.O.; Häggi, C.; Zabel, M.; Portilho-Ramos, R.C.; Schefuß, E.; Crivellari, S.; Wefer, G. 2017.
Different Precipitation Patterns Across Tropical South America During Heinrich and DansgaardOeschger Stadials. Quaternary Science Reviews, 177, 1-9. 